\title{
Robust Fault Diagnosis and Adaptive Parameter Identification for Single Phase Transformerless Inverters
}

\author{
Jian Li, Xinxin Guo, and Bo Li (iD \\ School of Automation Engineering, Northeast Electric Power University, Jilin 132012, China \\ Correspondence should be addressed to Bo Li; libo@neepu.edu.cn
}

Received 29 January 2018; Revised 27 June 2018; Accepted 31 July 2018; Published 13 August 2018

Academic Editor: George A. Papakostas

Copyright (C) 2018 Jian Li et al. This is an open access article distributed under the Creative Commons Attribution License, which permits unrestricted use, distribution, and reproduction in any medium, provided the original work is properly cited.

\begin{abstract}
The paper presents the theoretical analysis and simulation verification of robust fault diagnosis and adaptive parameter identification for single phase transformerless inverters. The fault diagnosis is composed of two parts, fault detection and fault identification. In the fault detection part, a Luenberger observer is designed to realize the detection of faults. Then, we apply a bank of observers to identify the location of faults. Meanwhile, the fault identification observers based estimation along with a gradient descent algorithm are also used in the parameter identification to estimate the actual values of components in a single phase transformerless inverter. Not only we develop the design methodology for the robust fault diagnosis and adaptive parameter identifier but also we present simulation results. The simulation results show the effectiveness of fault diagnosis and the accurate tracking of changes in component parameters for a wide range.
\end{abstract}

\section{Introduction}

The reliability of power electronics systems is increasingly important in many applications: industry, transportation, electric power, and so on. Its effective operation directly affects the security and stability of the whole system. Therefore, fault diagnosis for power electronics systems becomes particularly significant, which can timely detect and eliminate the potential faults and guarantee the stable operation of the system. In recent years, fault diagnosis has received the considerable attentions of the scholars; its theory and technique have also achieved rapid development [1]. Many diagnosis methods are proposed, such as model-based method, expertsystem-based method, and neural-network-based method. For these methods, there have been great quantity literatures, for instance, the energy equipment [2], the major aspects of power electronics reliability [3-5], and a fast fault diagnosis method for dc-dc converters [6]. We can conclude that it has become very important to diagnose faults at their very inception in order to avoid catastrophic effect in the system and heavy financial losses.

There are a variety of power electronics applying adaptive parameter identification including single-stage boost inverter
[7], ac-dc converters [8], and dc-dc converters [9-11]. For instance, real-time system identification [12], fault diagnosis $[13,14]$, and adaptive predictive control systems $[15,16]$ have been investigated. In general, the techniques of adaptive parameter identification have been deeply studied $[17,18]$. Most of the algorithms for parameter identification [19] estimate the values of system parameters by comparing measurements from the physical system with a model structure in a real-time and online manner. It is confirmed that the accurate parameter estimation can be achieved by classical algorithm for the slow-varied systems when the sample frequency is much lower than the frequency of switching [20, 21]. Nevertheless, the classical methodologies have certain characteristics which limit the effectiveness of parameter identification [22]. The limitations including the failure to track the fast parameter changes [23], only one parameter at a time [24], and high computation hardware requirements and so on.

It is known from the above that the traditional fault diagnosis methods are usually applied to general switched systems; that is to say they are hardly used to power electronics systems. That motivates us to study the fault diagnosis of power electronics. Comparing with the conventional power 
detection techniques, the fault diagnosis method of the switched system has many superiorities. We apply it to a single phase transformerless inverter.

In this paper, a reasonable robust fault diagnosis scheme and a robust adaptive parameter identification method are presented. Firstly, the fault model of a single phase transformerless inverter, which accurately captures the dynamics characteristics of the inverter, is considered. Subsequently, we design a robust fault detection observer to output residuals between the practical output of the system and the estimated output. The residual evaluation function is calculated to judge the fault. Moreover, for identifying the fault, a series of fault identification observers are designed to ensure the location of failure. Meanwhile, an improved adaptive parameter identification method is proposed. We adapt a generalized gradient descent algorithm to track and estimate the fault parameter.

The remainder of the paper is organized as follows. Section 2 presents the problem formulation. Section 3 presents the fault detection. The fault identification and adaptive parameter estimation are described in Section 4. Sections 5 and 6 present the thresholds computation and the simulation study. The last section concludes this paper.

Notation. For a matrix $A, A^{T}$ denotes its transpose. For a symmetric matrix, $A>0(A \geq 0)$ and $A<0$ $(A \leq 0)$ denote positive-definiteness (positive semidefinite matrix) and negative-definiteness (negative semidefinite matrix), respectively. The Hermitian part of a square matrix $M$ is denoted by $\mathrm{He}(M):=M+M^{T} . X_{i}$ represents a matrix in which all elements are one with appropriate dimensions. The symbol $*$ within a matrix represents the symmetric entries. 0 and $I$ denote the zero matrix and unit matrix with appropriate dimensions.

\section{Problem Formulation}

In this section, we present the modeling framework of a single phase transformerless inverter and describe kinds of fault cases in the inverter. Firstly, linear-switched model of the single phase transformerless inverter is described. Secondly, its faults are formulated. Finally, our design objective and the scheme of the proposed method are presented.

2.1. System Configuration. The single phase transformerless inverter is shown in Figure 1. Using a similar modeling approach to [25], it can be modeled by a linear-switched system as follows:

$$
\begin{aligned}
& \dot{x}(t)=A_{\sigma(t)} x(t)+B_{\sigma(t)} u(t), \\
& y(t)=C x(t)
\end{aligned}
$$

In the upper equation, $x(t), y(t)$, and $u(t)$ are the state vector, output vector, and input vector, respectively; $\sigma(t)$ is the continuous-time switching signal. $A_{\sigma(t)}, B_{\sigma(t)}$, and $C$ are the collection of state space models, which vary as a function of $\sigma(t)$. Table 1 shows the possible for the switching signal $\sigma(t)$, $s_{i}=0$ if switch $S W_{i}$ is open, and $S_{i}=1$ if $S W_{i}$ is close, $i \epsilon$ $[1,2,3,4]$.
TABle 1: Possible open/close switch positions for $S W_{i}$.

\begin{tabular}{lll}
\hline$\sigma(t)$ & 1 & 2 \\
\hline$s_{1}$ & 1 & 0 \\
$s_{2}$ & 0 & 1 \\
$s_{3}$ & 0 & 1 \\
$s_{4}$ & 1 & 0 \\
\hline
\end{tabular}

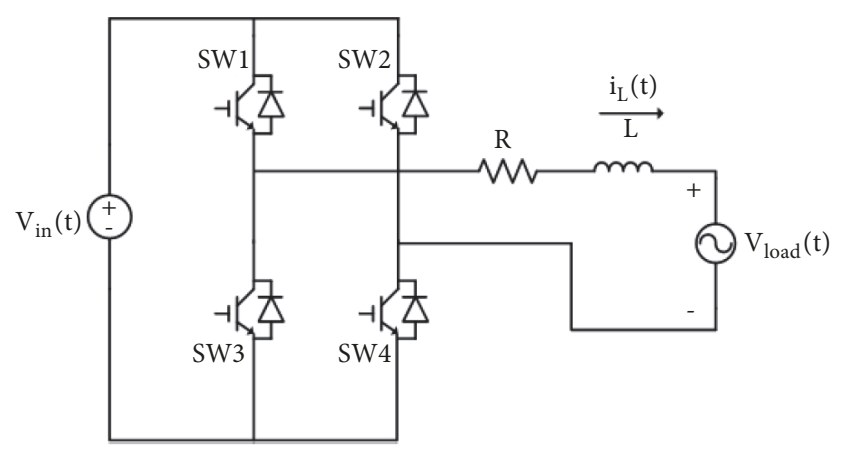

FIGURE 1: Single phase transformerless inverter.

Example 1. Consider a power electronic system shown in Figure 1; the linear-switched model of a single phase transformerless inverter is given by

$$
\begin{aligned}
x(t) & =y(t)=\left[i_{L}(t)\right], \\
u(t) & =\left[\begin{array}{c}
V_{\text {in }}(t) \\
V_{\text {load }}(t)
\end{array}\right], \\
A_{\sigma(t)} & =\left[-\frac{R}{L}\right], \\
B_{\sigma(t)} & =\left[\frac{s_{1}-s_{2}}{L}-\frac{1}{L}\right], \\
C & =[1]
\end{aligned}
$$

2.2. Fault Model. Next, we model the component fault dynamics in the power electronic inverter. For the system of Figure 1, we consider the inductance $L$ fault and the resistance $R$ fault. Once the components experience faults, the matrix parameters $A_{\sigma(t)}$ and $B_{\sigma(t)}$ are not the original values. Meanwhile, the state $x(t)$ and the output $y(t)$ are also influenced. We describe the system with these kinds of faults as follows:

$$
\begin{aligned}
& \dot{x}(t)=A_{\sigma(t)} x(t)+B_{\sigma(t)} u(t)+H f_{p}(t), \\
& y(t)=C x(t)
\end{aligned}
$$

where $H=I$ with appropriate dimensions; $p=1, \ldots, \tau$, $\tau$ is the number of fault types; $f_{p}(t)$ is the fault invertor when the $p_{t h}$ fault happens.

Example 2. Consider a fault in the inductance $L$ that the value of the inductance $L$ changes to $L-\Delta L$, where $\Delta L$ describes 
TABLE 2: The fault signal.

\begin{tabular}{llll}
\hline & Parameter & Fault events & Fault signal \\
\hline $\mathrm{p}=1$ & $\theta_{11}=\frac{1}{L}$ & Lchanges to $L-\Delta L$ & $f_{1}(t)=\left[-\frac{R \Delta L}{L(L-\Delta L)} i_{L}(t)+\frac{\left(s_{1}-s_{2}\right) \Delta L}{L(L-\Delta L)} V_{\text {in }}(t)-\frac{\Delta L}{L(L-\Delta L)} i_{\text {load }}(t)\right]$ \\
\hline $\mathrm{p}=2$ & $\theta_{21}=R$ & Rchanges to $R-\Delta R$ & $f_{2}(t)=\left[-\frac{\Delta R}{L} i_{L}(t)\right]$ \\
\hline $\mathrm{p}=3$ & $\theta_{31}=\frac{1}{L}$ & Lchanges to $L-\Delta L$ & $f_{3}(t)=\left[\frac{1}{L(L-\Delta L)} i_{L}(t)+\frac{\Delta L\left(s_{1}-s_{2}\right)}{L(L-\Delta L)} V_{\text {in }}(t)-\frac{\Delta L}{L(L-\Delta L)} V_{\text {load }}(t)\right]$ \\
\hline
\end{tabular}

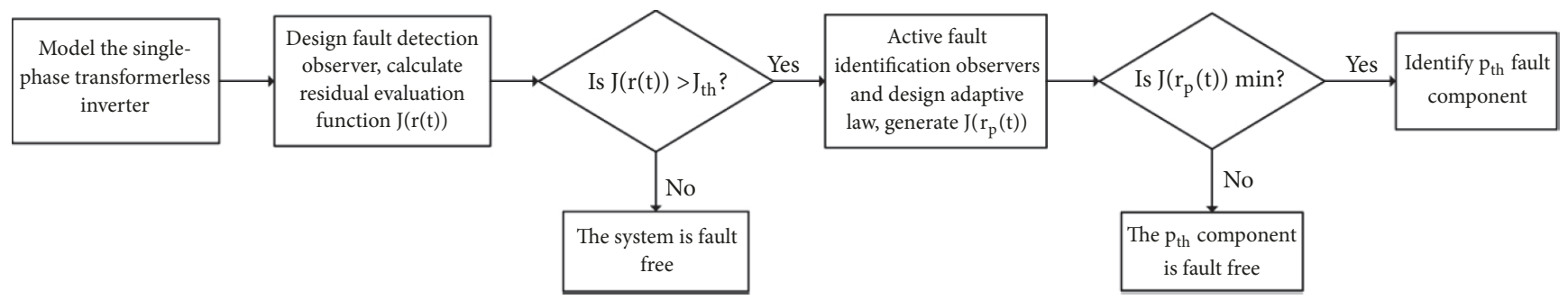

FIGURE 2: Scheme of the proposed method.

the magnitude of the fault. Thus, the system dynamics can be described as

$$
\dot{x}(t)=A_{\sigma(t)} x(t)+B_{\sigma(t)} u(t)
$$

where $A_{\sigma(t)}=[-R /(L-\Delta L)]$ and $B_{\sigma(t)}=\left[\left(s_{1}-s_{2}\right) /(L-\right.$ $\Delta L)-1 /(L-\Delta L)]$. Then, the upper equation can be rewritten as

$$
\dot{x}(t)=A_{\sigma(t)} x(t)+B_{\sigma(t)} u(t)+H f_{1}(t)
$$

where $f_{1}(t)=\left[-(R \Delta L / L(L-\Delta L)) i_{L}(t)+\left(\left(s_{1}-s_{2}\right) \Delta L /\right.\right.$ $\left.L(L-\Delta L)) V_{\text {in }}(t)-(\Delta L / L(L-\Delta L)) i_{\text {load }}(t)\right], H=[1]$.

A similar development follows for this case; we can describe other kinds of faults in Table 2, where $\theta_{p i}$ represents the parameter of $i_{t h}$ component when the $p_{t h}$ fault type occurs.

2.3. Design Objective. Consider the single phase transformerless inverter system modeled as switched system (3); the objective is to design a fault detection observer and a bank of fault identification observers with adaptive parameter identification. The scheme of the proposed method is involved in Figure 2.

2.3.1. Fault Detection. The fault detection observer is designed for an arbitrary switching signal and generates a residual signal $r(t)$ to satisfy the robust performance. When a fault occurs, the residual signal $r(t)$ is sensitive and changes rapidly. Then, the residual evaluation function $J(r(t))$ is larger than a predefined threshold $J_{t h}$; the fault could be detected as quickly as possible.

2.3.2. Fault Identification and Adaptive Parameter Identification. Once the fault has been detected, the fault identification determines the location of the component where the fault originated; then several fault identification observers work to estimate the specified fault components. If the $p_{t h}$ estimated fault component matches the occurred fault, the output of the $p_{t h}$ fault identification observer will converge to system (3), and the residual signal $r_{p}(t)$ between them will converge to zero. If not, there exists the error between the output of the $p_{t h}$ fault identification observer and system (3). Hence, we compare the evaluation function $J\left(r_{p}(t)\right)$ to identify the location of the fault. Moreover, an adaptive law is designed to estimate accurately the values of the components.

\section{Fault Detection}

This section proposes the design methodology for the fault detection. We design a Luenberger observer to detect the system fault [26].

The linear-switched fault detection observer is given by the following equations:

$$
\begin{aligned}
\dot{\hat{x}}(t) & =A_{\sigma(t)} \widehat{x}(t)+B_{\sigma(t)} u(t)+\mathscr{L} r(t), \\
r(t) & =y(t)-\widehat{y}(t), \\
\hat{y}(t) & =C \hat{x}(t)
\end{aligned}
$$

where $\widehat{x}(t)$ is the state estimation vector; $\mathscr{L}$ is the observer gain matrix; and $r(t)$ is the observer residual vector which is the difference between the actual output $y(t)$ and the estimated output $\widehat{y}(t)$.

For fault detection, it is not necessary to estimate the fault $f_{p}(t)$ in (3). It is interested in the fault signal of a certain frequency interval. Thus, we can formulate the estimated fault as the weighted fault $\widehat{f}(t)=W_{f}(s) f_{p}(t)$, where $W_{f}(s)$ is a 
given stable weighted matrix. A minimal realization of $\widehat{f}(t)=$ $W_{f}(s) f_{p}(t)$ is supposed to be

$$
\begin{aligned}
& \dot{x}_{w}(t)=A_{w} x_{w}(t)+B_{w} f_{p}(t), \\
& \hat{f}_{p}(t)=C_{w} x_{w}(t)+D_{w} f_{p}(t)
\end{aligned}
$$

where $x_{w}(t) \in \mathscr{R}^{n}$ is the state of the weighted fault, $f_{p}(t) \in \mathscr{R}^{m}$ is the original fault, and $\widehat{f}_{p}(t) \in \mathscr{R}^{m}$ is the weighted fault. $A_{w}, B_{w}, C_{w}$, and $D_{w}$ are known constant matrices.

Denote $x_{e}(t)=x(t)-\widehat{x}(t), \tilde{x}(t)=\left[x_{e}^{T}(t) x_{w}^{T}(t)\right]^{T}$, and $r_{e}(t)=r(t)-\widehat{f}_{p}(t)$. The augmented switched system is described as

$$
\begin{aligned}
& \dot{\tilde{x}}(t)=\widetilde{A} \tilde{x}(t)+\widetilde{B} f_{p}(t), \\
& r_{e}(t)=\widetilde{C} \widetilde{x}(t)+\widetilde{D} f_{p}(t)
\end{aligned}
$$

where

$$
\begin{aligned}
& \widetilde{A}=\left[\begin{array}{cc}
A_{\sigma(t)}-L C & 0 \\
0 & A_{w}
\end{array}\right], \\
& \widetilde{B}=\left[\begin{array}{l}
H \\
B_{w}
\end{array}\right] \\
& \widetilde{C}=\left[\begin{array}{ll}
C & -C_{w}
\end{array}\right], \\
& \widetilde{D}=\left[-D_{w}\right]
\end{aligned}
$$

Now, the frameworks of fault detection design can be formulated as follows: considering system (8), dynamic observer gains are obtained for an arbitrary switching signal. Then system (8) satisfies the following requirements:

(i) System (8) is asymptotically stable.

(ii) For detection objective, the effects from the faults to the residual error signal $r_{e}(t)$ are minimized; i.e., the FD observer satisfies the following index with

$$
\int_{0}^{\infty} r_{e}^{T}(t) r_{e}(t) d t<\gamma^{2} \int_{0}^{\infty} f^{T}(t) f(t) d t
$$

For obtaining the gain of the fault detection observer and satisfying the performance (10), the following theorem is given to design the fault detection observer.

Theorem 3. Given the constant $a_{1 \sigma}, a_{2 \sigma}, a_{3 \sigma}$, if there exist matrix variables $W_{1}, W_{2 \sigma}, W_{3 \sigma}, W_{4 \sigma}, W_{5 \sigma}, \mathbf{L}, M, P=\left[\begin{array}{cc}P_{1} & P_{2} \\ * & P_{3}\end{array}\right]>$ 0 satisfying the inequalities

$$
\left[\begin{array}{cccc}
\Phi_{11} & \Phi_{12} & \Phi_{13} & 0 \\
* & \Phi_{22} & \Phi_{23} & \mathscr{C}^{T} \\
* & * & -\gamma^{2} I & \mathscr{D}^{T} \\
* & * & * & -I
\end{array}\right]<0
$$

where

$$
\begin{aligned}
& \Phi_{11}=\left[\begin{array}{cc}
-H e\left(W_{1}\right) & -a_{1 \sigma} W_{1}-W_{2 \sigma}^{T} \\
* & -H e\left(W_{3 \sigma}\right)
\end{array}\right], \\
& \Phi_{12}=\left[\begin{array}{cc}
P_{1}-a_{2 \sigma} W_{1}+W_{1}^{T} A_{\sigma}-\mathfrak{\Omega} C & P_{2}-a_{3 \sigma} W_{1}+W_{2 \sigma}^{T} A_{w} \\
P_{2}^{T}-W_{4 \sigma}+a_{1 \sigma} W_{1}^{T} A_{\sigma}-a_{1 \sigma} \mathcal{\Omega} C & P_{3}-W_{5 \sigma}+W_{3 \sigma}^{T} A_{w}
\end{array}\right], \\
& \Phi_{13}=\left[\begin{array}{c}
W_{1}^{T} H+W_{2 \sigma}^{T} B_{w} \\
a_{1 \sigma} W_{1}^{T} H+W_{3 \sigma}^{T} B_{w}
\end{array}\right], \\
& \Phi_{22} \\
& =\left[\begin{array}{c}
a_{2 \sigma} H e\left(A_{\sigma(t)}^{T} W_{1}-\mathfrak{\Omega} C\right) \\
* \\
*
\end{array} W_{4 \sigma}^{T} A_{w}+a_{3 \sigma} A_{\sigma(t)}^{T} W_{1}-a_{3 \sigma} C^{T} \mathbf{2}^{T}\right. \\
& \Phi_{23}=\left[\begin{array}{c}
\left.a_{2 \sigma} W_{1}^{T} H+W_{4 \sigma}^{T} A_{w}\right) \\
a_{3 \sigma} W_{1}^{T} H+W_{5 \sigma}^{T} B_{w}
\end{array}\right],
\end{aligned}
$$

then system (8) under arbitrary switching is asymptotically stable and guarantees the robust performance (10). Moreover, if (11) is feasible, then the observer gains in form of (6) can be given by $\mathscr{L}=W_{1}^{-1} \mathfrak{\Omega}$.

Proof. Firstly, consider the stability for system (8), we rewrite the system as $\dot{\tilde{x}}(t)=\widetilde{\mathscr{A}} \tilde{x}(t)$ when $f(t)=0$ and choose the common Lyapunov functions: $V(\tilde{x}(t))=\tilde{x}^{T}(t) P \tilde{x}(t)$. Then it has

$$
\dot{V}_{\sigma}(\tilde{x}(t))=\tilde{x}^{T}(t)\left(\widetilde{\mathscr{A}}^{T} P+P \widetilde{\mathscr{A}}\right) \tilde{x}
$$

We consider the following performance index: $\Gamma(t)=$ $\int_{0}^{t}\left(r_{e}^{T}(\tau) r_{e}(\tau)-\gamma^{2} f^{T}(\tau) f(\tau)\right) d \tau$. For any nonzero $f(t) \in$ $l_{2}[0, \infty)$ and the under zero-initial condition, we have

$\Gamma(t)$

$$
\begin{aligned}
= & \int_{0}^{t}\left(r_{e}^{T}(\tau) r_{e}(\tau)-\gamma^{2} f^{T}(\tau) f(\tau)+\dot{V}(\tilde{x}(\tau))\right) d \tau \\
& -V(\tilde{x}(t)) \\
\leq & \int_{0}^{t}\left(r_{e}^{T}(t) r_{e}(t)-\gamma^{2} f^{T}(t) f(t)+\dot{V}_{\sigma}(\tilde{x}(\tau))\right) d \tau
\end{aligned}
$$

It notes that

$$
\begin{aligned}
r_{e}^{T} & (\tau) r_{e}(\tau)-\gamma^{2} f^{T}(\tau) f(\tau)+\dot{V}_{\sigma}(\tilde{x}(\tau)) \\
= & {\left[\begin{array}{l}
\tilde{x}(\tau) \\
f(\tau)
\end{array}\right]^{T} \Lambda\left[\begin{array}{l}
\tilde{x}(\tau) \\
f(\tau)
\end{array}\right] }
\end{aligned}
$$

where

$$
\Lambda=\left[\begin{array}{cc}
\widetilde{\mathscr{A}}^{T} P+P \widetilde{\mathscr{A}}+\widetilde{\mathscr{C}}^{T} \widetilde{\mathscr{C}} & \widetilde{\mathscr{B}}^{T} P+\widetilde{\mathscr{C}}^{T} \widetilde{\mathscr{D}} \\
* & -\gamma^{2} I+\widetilde{\mathscr{D}}^{T} \widetilde{\mathscr{D}}
\end{array}\right]
$$

Thus, if $\Lambda<0$, it follows form (13) that $\dot{V}_{\sigma}(\tilde{x}(t))<$ 0 , which implies that $V(\tilde{x}(t))$ converges to zero as $t \longrightarrow$ $\infty$. The switched system (8) under arbitrary switching is 
asymptotically stable. Moreover, it also implies $\Gamma(t)<0$. Then, it has the robust performance (10).

It notes that $\Lambda$ can be rewritten as

$$
\begin{aligned}
& {\left[\begin{array}{lll}
\widetilde{\mathscr{A}}^{T} & I & 0 \\
\widetilde{\mathscr{B}}^{T} & 0 & I
\end{array}\right]\left[\begin{array}{ccc}
0 & P & 0 \\
P & \widetilde{\mathscr{C}}^{T} \widetilde{\mathscr{C}} & \widetilde{\mathscr{C}}^{T} \widetilde{\mathscr{D}} \\
0 & \widetilde{\mathscr{D}}^{T} \widetilde{\mathscr{C}} & -\gamma^{2} I+\widetilde{\mathscr{D}}^{T} \widetilde{\mathscr{D}}
\end{array}\right]\left[\begin{array}{cc}
\widetilde{\mathscr{A}} & \widetilde{\mathscr{B}} \\
I & 0 \\
0 & I
\end{array}\right]} \\
& \quad<0
\end{aligned}
$$

By Projection theorem, (17) is equivalent to

$$
\begin{aligned}
& {\left[\begin{array}{ccc}
0 & P & 0 \\
P & \widetilde{\mathscr{C}}^{T} \widetilde{\mathscr{C}} & \widetilde{\mathscr{C}}^{T} \widetilde{\mathscr{D}} \\
0 & \widetilde{\mathscr{D}}^{T} \widetilde{\mathscr{C}} & -\gamma^{2} I+\widetilde{\mathscr{D}}^{T} \widetilde{\mathscr{D}}
\end{array}\right]} \\
& \quad+H e\left(\left[\begin{array}{c}
-I \\
\widetilde{\mathscr{A}}^{T} \\
\widetilde{\mathscr{B}}^{T}
\end{array}\right]\left[\begin{array}{lll}
\mathscr{W}_{1} & \mathscr{W}_{2} & 0
\end{array}\right]\right)<0
\end{aligned}
$$

where $\mathscr{W}_{1} \mathscr{W}_{2}$ are the matrix variables of appropriate dimensions, and let $\mathscr{W}_{1}=\left[\begin{array}{cc}W_{1} & a_{1 \sigma} W_{1} \\ W_{2 \sigma} & W_{3 \sigma}\end{array}\right]$ and $\mathscr{W}_{2}=$ $\left[\begin{array}{cc}a_{2 \sigma} W_{1} & a_{3 \sigma} W_{1} \\ W_{4 \sigma} & W_{5 \sigma}\end{array}\right]$. By denoting $\mathfrak{Q}=W_{1}^{T} \mathscr{L}$ and applying Schur complement formula, and after some matrix manipulation, (18) becomes (11).

\section{Fault Identification and Adaptive Parameter Identification}

In this section, the fault identification and an improved adaptive parameter identification method [27] are presented for the single transformerless inverter. After a fault is detected, a bank of fault identification observers is activated to recognize the location of fault. Each observer in the bank corresponds to an inverter component to real time estimate the numerical parameter values. When the certain component has a fault, its residual signal generated by the corresponding observer is minimum of all the evaluation functions for the residual vectors generated by the other observers.

Now we assume that the fault is detected; the fault identification observers are activated. As Table 2, the faults exist in inductance $L$, resistance $R$ or inductance $L$ and resistance $R$. We denote the parameters $\theta_{11}=1 / L, \theta_{21}=R$, $\theta_{31}=1 / L, \theta_{32}=R$; then we design two observers. We assume that the parameter $\theta_{p i}$ has a fault; the switched system (1) can be rewritten as

$$
\begin{aligned}
& \dot{x}(t)=A_{\sigma(t)}\left(\theta_{p i}\right) x(t)+B_{\sigma(t)}\left(\theta_{p i}\right) u(t), \\
& y(t)=C x(t)
\end{aligned}
$$

where $A_{\sigma(t)}\left(\theta_{p i}\right)$ and $B_{\sigma(t)}\left(\theta_{p i}\right)$ are the system matrices which use $\theta_{p i}$ in $A_{\sigma(t)}$ and $B_{\sigma(t)}$.

The parameter values of the system contained in matrices $A_{\sigma(t)}$ and $B_{\sigma(t)}$ should be precisely known for accurate state estimation of system. However, $A_{\sigma(t)}$ and $B_{\sigma(t)}$ are not precisely known when the values of components change and cannot be measured accurately because of faults. Consider this case; the joint state and parameter estimation is encapsulated by the following equations:

$$
\begin{aligned}
\dot{\hat{x}}_{p}(t)= & A_{\sigma(t)}\left(\widehat{\theta}_{p i}\right) x(t)+B_{\sigma(t)}\left(\widehat{\theta}_{p i}\right) u(t) \\
& +\left(\mathscr{L}_{p}+\Gamma(t) T_{p} \Gamma^{T}(t) C^{T}\right) r_{p}(t), \\
\hat{y}_{p}(t)= & C \widehat{x}(t), \\
r_{p}(t)= & y(t)-\hat{y}_{p}(t)
\end{aligned}
$$

where $\widehat{x}_{p}(t)$ and $\widehat{y}_{p}(t)$ are the $p_{t h}$ observer state and output, respectively. $\widehat{\theta}_{p i}$ is the estimate signals of $\theta_{p i}$. T $\epsilon$ $R^{n \times n}$ is a chosen symmetric positive-definite matrix such that the dynamics of $\dot{\hat{\theta}}_{p i}(t)$ evolve much slower than the state dynamics $\widehat{x}(t) . n$ is the dimension of $\dot{\hat{\theta}}_{p i}(t) . \Gamma(t)$ is the additive vector variable. $r_{p}(t)$ is the residual signal of fault identification observer, and $\mathscr{L}_{p}$ is the observer gain.

Denote $\tilde{x}_{p}(t)=x(t)-\widehat{x}_{p}(t)$ and $\widetilde{\theta}_{p i}=\theta_{p i}-\widehat{\theta}_{p i}$. Then, the error system is described as

$$
\begin{aligned}
\dot{\tilde{x}}_{p}(t)= & {\left[A_{\sigma(t)}-\mathscr{L}_{p} C\right] \tilde{x}_{p}(t)+\Psi_{p}(t) \tilde{\theta}_{p i} } \\
& -\Gamma(t) T_{p} \Gamma^{T}(t) C^{T} r_{p}(t)
\end{aligned}
$$

where $\Psi_{p}(t)=\left(A_{\sigma(t)}\left(\theta_{p i}\right)-A_{\sigma(t)}\left(\widehat{\theta}_{p i}\right)\right) \widehat{x}_{p}(t)+\left(B_{\sigma(t)}\left(\theta_{p i}\right)-\right.$ $\left.B_{\sigma(t)}\left(\widehat{\theta}_{p i}\right)\right) u(t)$.

For obtaining the gain of the observer and realizing the adaptive parameter identification, the following theorem is given.

Theorem 4. If there exist matrix variables $\mathfrak{\Omega}_{p}, P>0$ satisfying the inequalities

$$
A_{\sigma(t)}^{T} P-C^{T} \mathbf{\Omega}_{p}^{T}+P A_{\sigma(t)}-\mathfrak{Q}_{p} C \leq 0
$$

then system (21) under arbitrary switching is asymptotically stable, and $\theta_{p i}$ is determined according to the adaptive law:

$$
\begin{aligned}
\dot{\hat{\theta}}_{p i}(t) & =T_{p} \Gamma^{T}(t) C^{T}\left(y(t)-\widehat{y}_{p}(t)\right), \\
\dot{\Gamma}(t) & =\left[A_{\sigma(t)}-\mathscr{L}_{p} C\right] \tilde{x}_{p}(t)+\Psi_{p}(t) \tilde{\theta}_{p i}
\end{aligned}
$$

Moreover, if (22) is feasible, then the fault identification observer gain in form of (20) can be given by $\mathscr{L}_{p}=P^{-1} \mathfrak{Q}_{p}$.

Proof. Similar to [28], the classical Lyapunov's direct method is adapted to analyze the stability of the system (21). According to this, we denote $\zeta(t)=\widetilde{x}_{p}(t)-\Gamma(t) \widetilde{\theta}_{p i}$ and choose the common Lyapunov function: $V(t)=\zeta^{T}(t) P \zeta(t)$. Then from the derivative of $V(t)$ along system (21), it has

$$
\begin{aligned}
& \dot{V}(t)=\zeta^{T}(t)\left[\left(A_{\sigma(t)}-\mathscr{L}_{p} C\right)^{T} P\right. \\
& \left.\quad+P\left(A_{\sigma(t)}-\mathscr{L}_{p} C\right)\right] \zeta(t)+2 \zeta^{T}(t)
\end{aligned}
$$




$$
\begin{aligned}
& \cdot P\left[\left(A_{\sigma(t)}-\mathscr{L}_{p} C\right) \Gamma(t) \tilde{\theta}_{p i}+\Psi_{p}(t) \tilde{\theta}_{p i}\right. \\
& \left.-\Gamma(t) T_{p} \Gamma^{T}(t) C^{T} r_{p}(t)-\dot{\Gamma}(t) \tilde{\theta}_{p i}-\Gamma(t) \dot{\tilde{\theta}}_{p i}\right]
\end{aligned}
$$

Choose the appropriate adaptive law that is presented in (23); it has

$$
\begin{aligned}
& \left(A_{\sigma(t)}-\mathscr{L}_{p} C\right) \Gamma_{p}(t) \widetilde{\theta}_{p i}+\Psi_{p}(t) \widetilde{\theta}_{p i} \\
& \quad-\Gamma(t) T_{p} \Gamma^{T}(t) C^{T} r_{p}(t)-\dot{\Gamma}(t) \widetilde{\theta}_{p i}-\Gamma(t) \dot{\tilde{\theta}}_{p i} \\
& \quad=0
\end{aligned}
$$

Hence, it has $\dot{V}(t)<\zeta^{T}(t)\left[\left(A_{\sigma(t)}-\mathscr{L}_{p} C\right)^{T} P+P\left(A_{\sigma(t)}-\right.\right.$ $\left.\left.\mathscr{L}_{p} C\right)\right] \zeta(t)$. If $(22)$ holds, $\dot{V}(t)<0$. Thus, when the chosen adaptive law is adapted, system (21) is asymptotically stable.

Example 5. Consider the failure of parameter $L$, according to Table 2: $\theta_{11}=1 / L$, the observer can be designed as

$$
\begin{aligned}
\dot{\hat{x}}_{L}(t)= & A_{\sigma(t)}\left(\widehat{\theta}_{L}\right) x(t)+B_{\sigma(t)}\left(\widehat{\theta}_{L}\right) u(t) \\
& +\left(\mathscr{L}_{L}+\Gamma(t) T_{L} \Gamma^{T}(t) C^{T}\right) r_{L}(t), \\
r_{L}(t)= & y(t)-C \hat{x}(t)
\end{aligned}
$$

where

$$
\begin{aligned}
A_{\sigma(t)}\left(\widehat{\theta}_{L}\right) & =\left[-R \widehat{\theta}_{L}\right], \\
B_{\sigma(t)}\left(\widehat{\theta}_{L}\right) & =\left[\begin{array}{ll}
\left(s_{1}-s_{2}\right) \widehat{\theta}_{L} & \widehat{\theta}_{L}
\end{array}\right]
\end{aligned}
$$

We can obtain the error system as

$$
\begin{aligned}
\dot{\tilde{x}}_{L}(t)= & {\left[A_{\sigma(t)}-\mathscr{L}_{L} C\right] \widetilde{x}_{L}(t)+\Psi_{L}(t) \widetilde{\theta}_{L} } \\
& -\Gamma(t) T_{L} \Gamma^{T}(t) C^{T} r_{L}(t)
\end{aligned}
$$

where

$$
\Psi_{L}(t)=\left[(s 1-s 2) V_{\text {in }}(t)-V_{\text {load }}(t)-R \hat{i_{L}}\right]
$$

According to Theorem 4 , the observer gain $\mathscr{L}_{L}$ can be calculated by conditions (22). The parameter $L$ can be precisely estimated according to the designed adaptive parameter identification.

\section{Thresholds Computation}

In this section, we compute the residual evaluation functions and determine the thresholds after obtaining the observer gains. The residual evaluation function $J(r(t))$ for the fault detection and the residual evaluation function $J\left(r_{p}(t)\right)$ for the fault identification can be chosen as

$$
\begin{gathered}
J(r(t))=\frac{1}{N} \int_{t-N}^{t} r(\tau)^{T} r(\tau) d \tau \\
J\left(r_{p}(t)\right)=\frac{1}{N} \int_{t-N}^{t} r_{p}^{T}(\tau) r_{p}(\tau) d \tau
\end{gathered}
$$

TABLE 3: Single phase transformerless inverter parameters.

\begin{tabular}{lc}
\hline 1Фinverterparameters & \\
$L$ & $5 m H \pm 20 \%$ \\
$R$ & $0.3 \Omega \pm 20 \%$ \\
\hline Operatingpoint & \\
$V_{\text {in }}(t)$ & $380 V_{d c}$ \\
$V_{c}(t)$ & $235 V_{r m s}$ \\
$R_{\text {load }}$ & $100 \Omega$ \\
\hline
\end{tabular}

where $N$ is the length of the evaluation window and $r(t)=y(t)-\widehat{y}(t)$ and $r_{p}(t)=y(t)-\widehat{y}_{p}(t)$ are residual signals for the fault detection observer and for the fault identification observers. The residual evaluation functions $J(r(t))$ and $J\left(r_{p}(t)\right)$ denote the average value from $r(t-$ $N)^{T} r(t-N)$ to $r(t)^{T} r(t)$ and from $r_{p}^{T}(t-N) r_{p}(t-N)$ to $r_{p}^{T}(t) r_{p}(t)$, respectively.

Fault Detection. We can set the threshold according to the selected evaluation function. The threshold $J_{t h}$ is selected in the no-fault condition. Thus, the other reasons will not let $J(r(t))$ exceed $J_{t h}$. The threshold is designed as

$$
J_{t h}=\sup _{f(k)=0} J(r(t)) .
$$

We can detect the faults by comparing $J(r(t))$ and $J_{t h}$ as follows:

$$
\begin{aligned}
& \|J(r(t))\| \leq J_{t h} \Longrightarrow \text { the system has fault, } \\
& \|J(r(t))\|>J_{t h} \Longrightarrow \text { the system has no fault. }
\end{aligned}
$$

Fault Identification. For the fault identification, we compare the evaluation function $J\left(r_{p}(t)\right)$ for all fault identification observers. As shown in Table 2, the inductance $L$ fault corresponds to parameter $p=1$, the resistance $R$ fault corresponds to parameter $p=2$, and the inductance $L$ and resistance $R$ all fault correspond to parameter $p=3$. The parameter $p$ is chosen to show the result of fault identification as

$$
\begin{gathered}
\min _{p} J\left(r_{p}(t)\right) \Longrightarrow \text { Parameter } p=h, h \in\{1,2,3\} \\
\Longrightarrow \text { identify that the } p_{t h} \text { fault has occurred. }
\end{gathered}
$$

\section{Simulation Study}

In this section, we first provide the design of the observers. Subsequently, a simulation study of fault diagnose and adaptive parameter identification for the single transformerless inverter is presented. The complete specifications of the invert parameters are presented in Table 3.

6.1. Design Result of the Observer. As Figure 1, we give the parameters of the single phase transformerless inverter system as Table 3. For the fault detection to estimate the fault $f_{p}(t)$, we choose a kind of high-pass filter as parameters $A_{w}=$ 


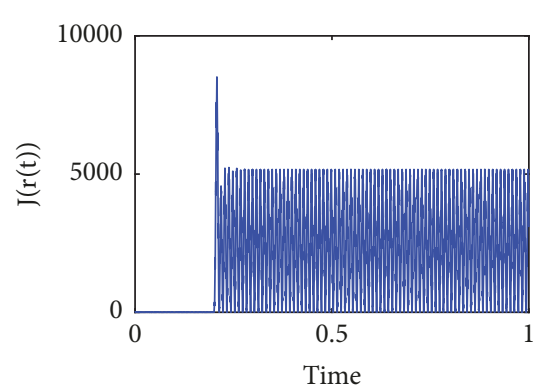

(a) $J(r(t))$ for FD observer

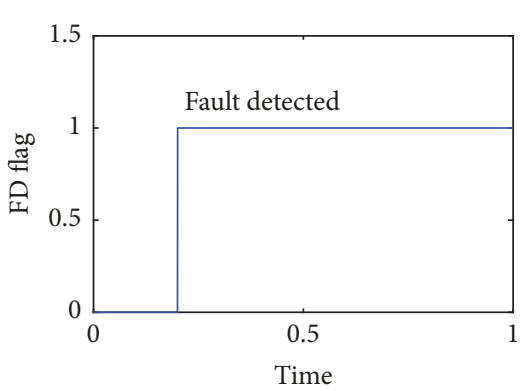

(b) FD flag

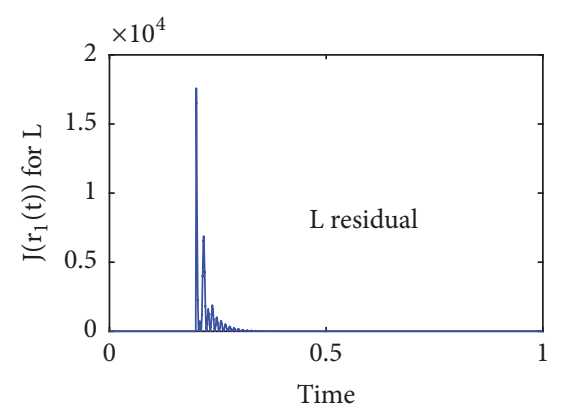

(c) $J\left(r_{1}(t)\right)$ for FI $L$ observer

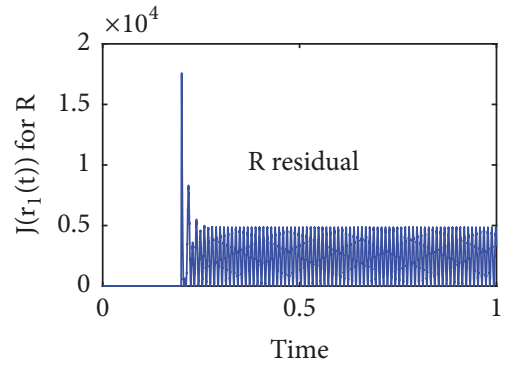

(d) $J\left(r_{1}(t)\right)$ for FI $R$ observer

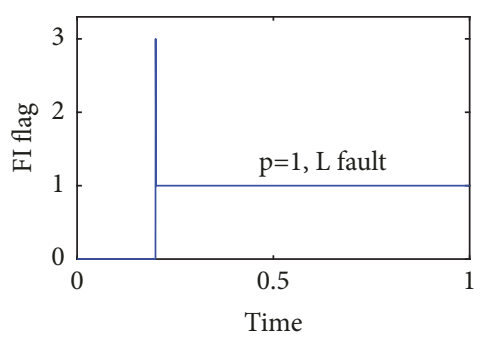

(e) FI flag

FIGURE 3: (a) and (b) are FD results as L experiences fault. (c), (d), and (e) are simulation results for FI as L experiences fault.

$[-1], B_{w}=[1], C_{w}=[1]$, and $D_{w}=[1]$ and give $a_{11}=1$, $a_{21}=30$, and $a_{31}=-0.3$. By solving the LMI (11), the fault detection observer gain is shown as

$$
\mathscr{L}=[31.6593]
$$

Consider the parameters in Table 2; two observers are designed for the inductance $L$ and the resistance $R$, respectively. We adapt the identical observer gain in two observers. By solving (22), the fault identification observer gain $\mathscr{L}$ is given as

$$
\mathscr{L}=[33.9073] .
$$

6.2. Fault Diagnosis Results. In this subsection, we simulate the residual signals for three types of faults by Piecewise Linear Electrical Circuit Simulation toolbox in MATLAB/Simulink environment.

6.2.1. Fault in the Inductance $L$. In this case, the fault occurs in the inductance $L$ which value reduces $33 \%$ at $t=0.2 s$. The residual evaluation function $J(r(t))$ is shown in Figure 3(a), which is quick over the threshold $J_{t h}=15$. Thus, the inductance fault has been injected as presented in Figure 3(b). The fault identification observers are activated after fault detection. As the output of these observers in Figures 3(c) and 3 (d), we can identify that the observer for the inductance $L$ matches the fault system and decide that the parameter $p$ of fault identification flag is one in Figure 3(e).

6.2.2. Fault in the Resistance $R$. The fault occurs in the resistance $R$ which value changes from $0.3 \Omega$ to $0.03 \Omega$. Similarly, Figures 4(a) and 4(b) present the fault detection observer residual and fault detection flag, respectively, for $R$ fault. From these results, the fault for resistance $R$ can be detected immediately at $t=0.2 \mathrm{~s}$ because the residual evaluation function $J(r(t))$ exceeds the threshold $J_{t h}$. Figure 4(e) shows that the fault identification flag $p=2$; in other words, the fault is identified by comparing the outputs of fault identification observers shown in Figures 4(c) and 4(d).

6.2.3. Fault in the Inductance $L$ and Resistance $R$. We consider the case that the inductance and resistance have all occurred faults, $L$ changes to $50 \%$, and $R$ increases to $0.4 \Omega$ at $t=0.2 s$. Figure 5(a) presents the residual evaluation function of fault detection; then the fault is detected as shown in Figure 5(b). The outputs of fault identification observers presented in Figures 5(c) and 5(d) all converge to zero. From the two figures, we can identify that the third kind fault occurs according to Table 2 as shown in Figure 5(e).

6.3. Adaptive Parameter Identification. Once a component fault happens, the value of this component changes. Hence, the section of fault identification and adaptive parameter identification design an adaptive law to reestimate the value of the component that has experienced fault. In this subsection, the simulation results of the adaptive parameter identification are presented. The simulation figures exhibit the tracking capability and convergence speed of the proposed parameter identification.

For inductance $L$, we considers the different degree fault from $67 \%$ to $10 \%$ when resistance $R$ does not vary. Figure 6 shows the results of this test. As shown, the fault value of inductance $L$ can be estimated accurately. As shown in Figure 7, we inject a different step perturbation in the 


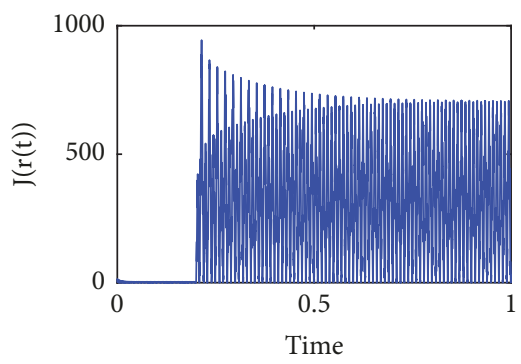

(a) $J(r(t))$ for FD observer

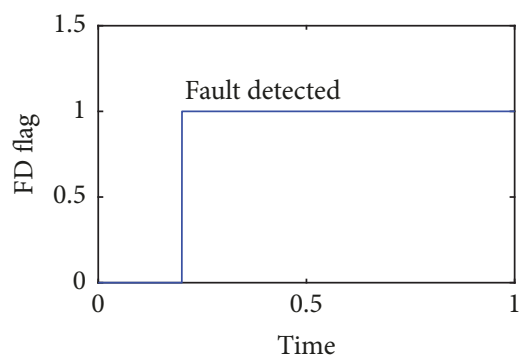

(b) FD flag

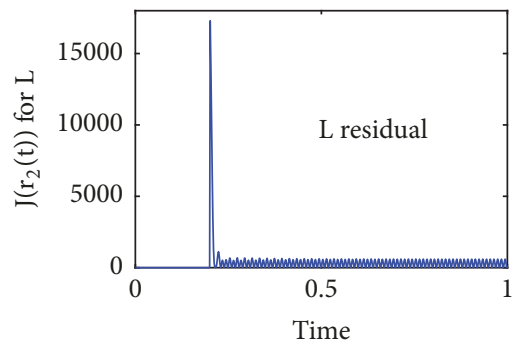

(c) $J\left(r_{2}(t)\right)$ for FI $L$ observer

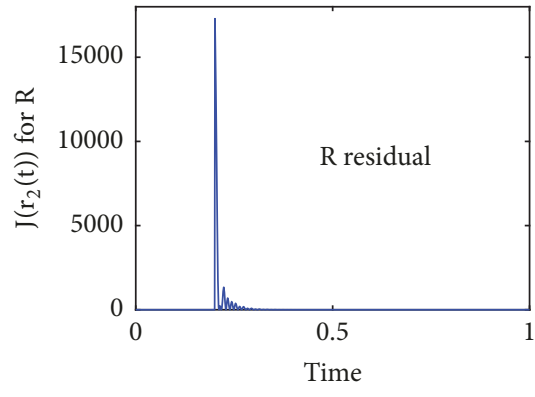

(d) $J\left(r_{2}(t)\right)$ for FI $R$ observer

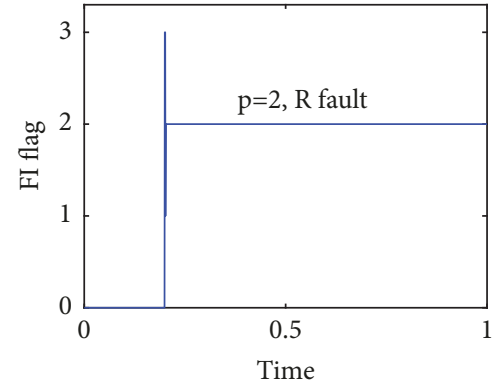

(e) FI flag

FIGURE 4: (a) and (b) are FD results as R experiences fault. (c), (d), and (e) are FI results as R experiences fault.

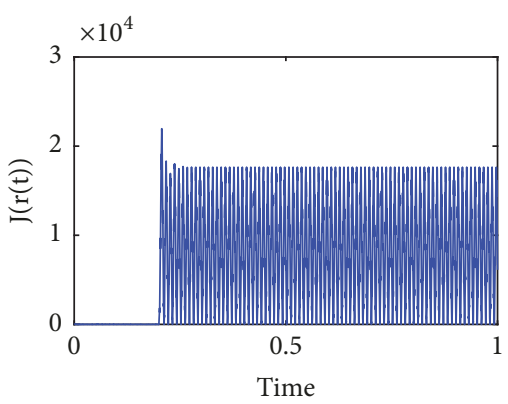

(a) $J(r(t))$ for FD observer

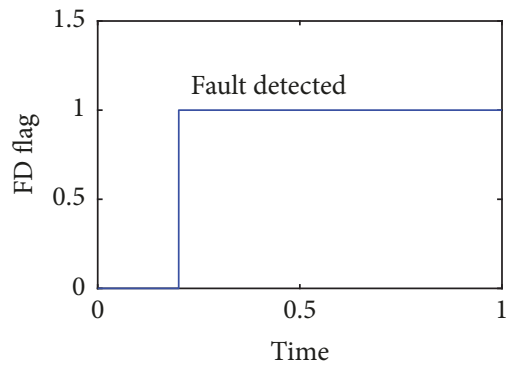

(b) FD flag

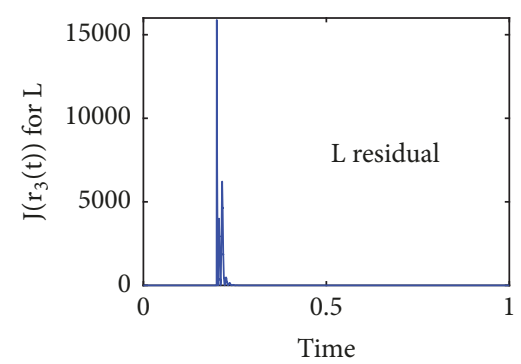

(c) $J\left(r_{3}(t)\right)$ for FI $L$ observer

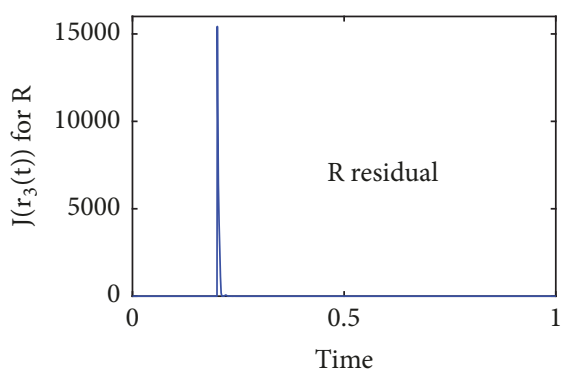

(d) $J\left(r_{3}(t)\right)$ for FI $R$ observer

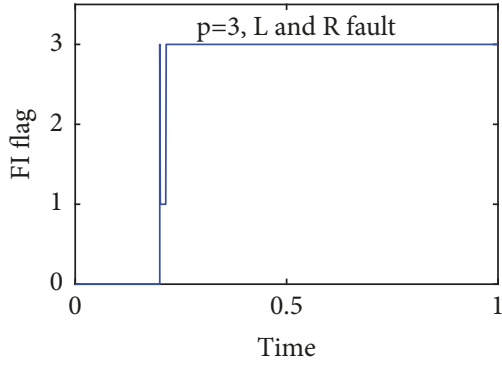

(e) FI flag

Figure 5: (a) and (b) are FD results as $L$ and $R$ all experience faults. (c), (d), and (e) are simulation results for FI as $L$ and $R$ all experience faults.

resistance $R: 10 \%, 33 \%, 50 \%$, and $67 \%$. The figure testifies the effectiveness of adaptive parameter identification. Finally, Figure 8 presents the tracking of multiple parameters inductance $L$ and resistance $R$. We can learn that the convergence for each parameter is independent of other parameter conditions from these simulation results.

\section{Conclusion}

This paper deals with the fault diagnosis and adaptive parameter identification of a single phase transformerless inverter. A fault detection observer and a bank of fault identification Luenberger observers, which are based 

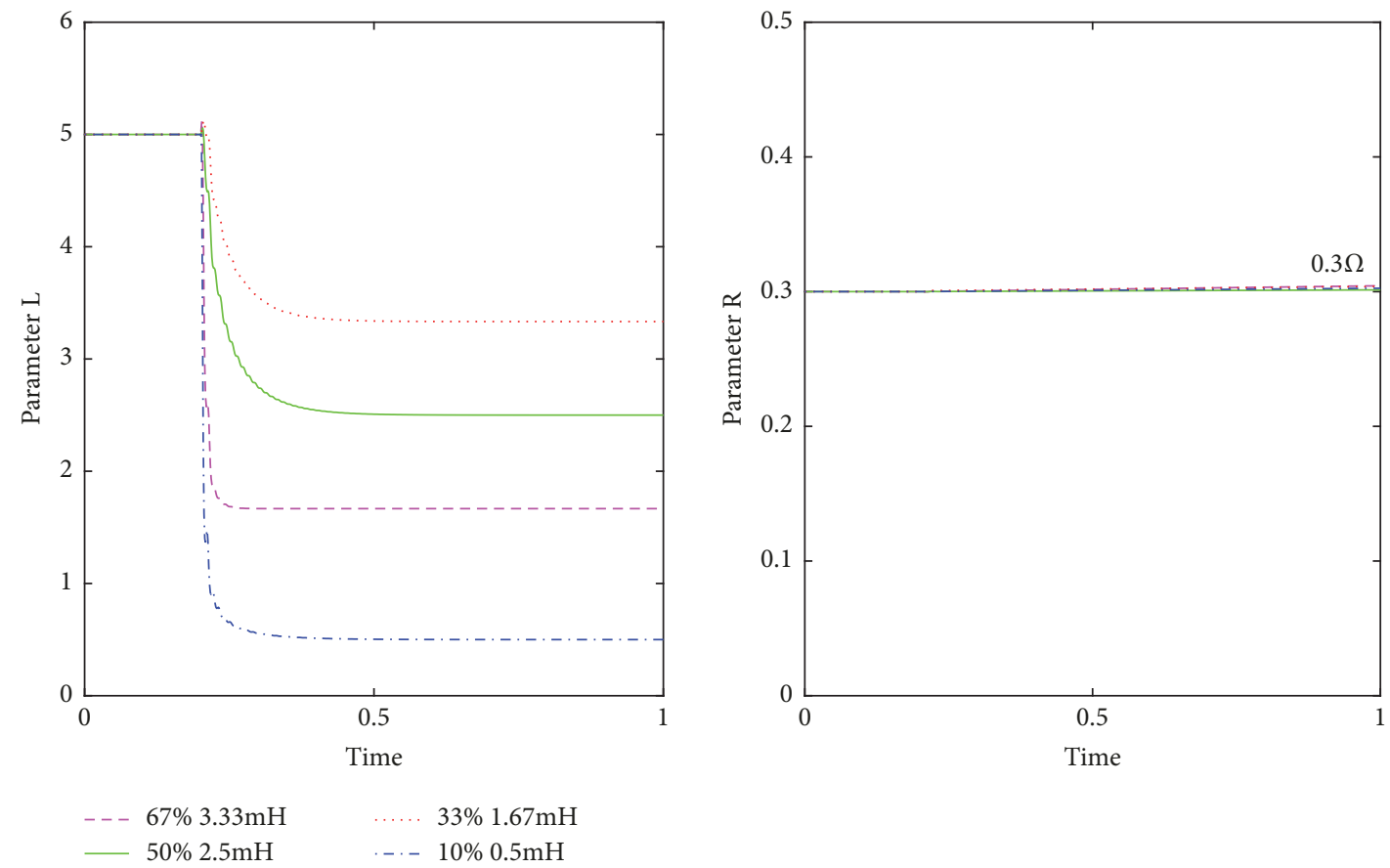

Figure 6: Parameter identification as $L$ changes from $67 \%$ to $10 \%$.
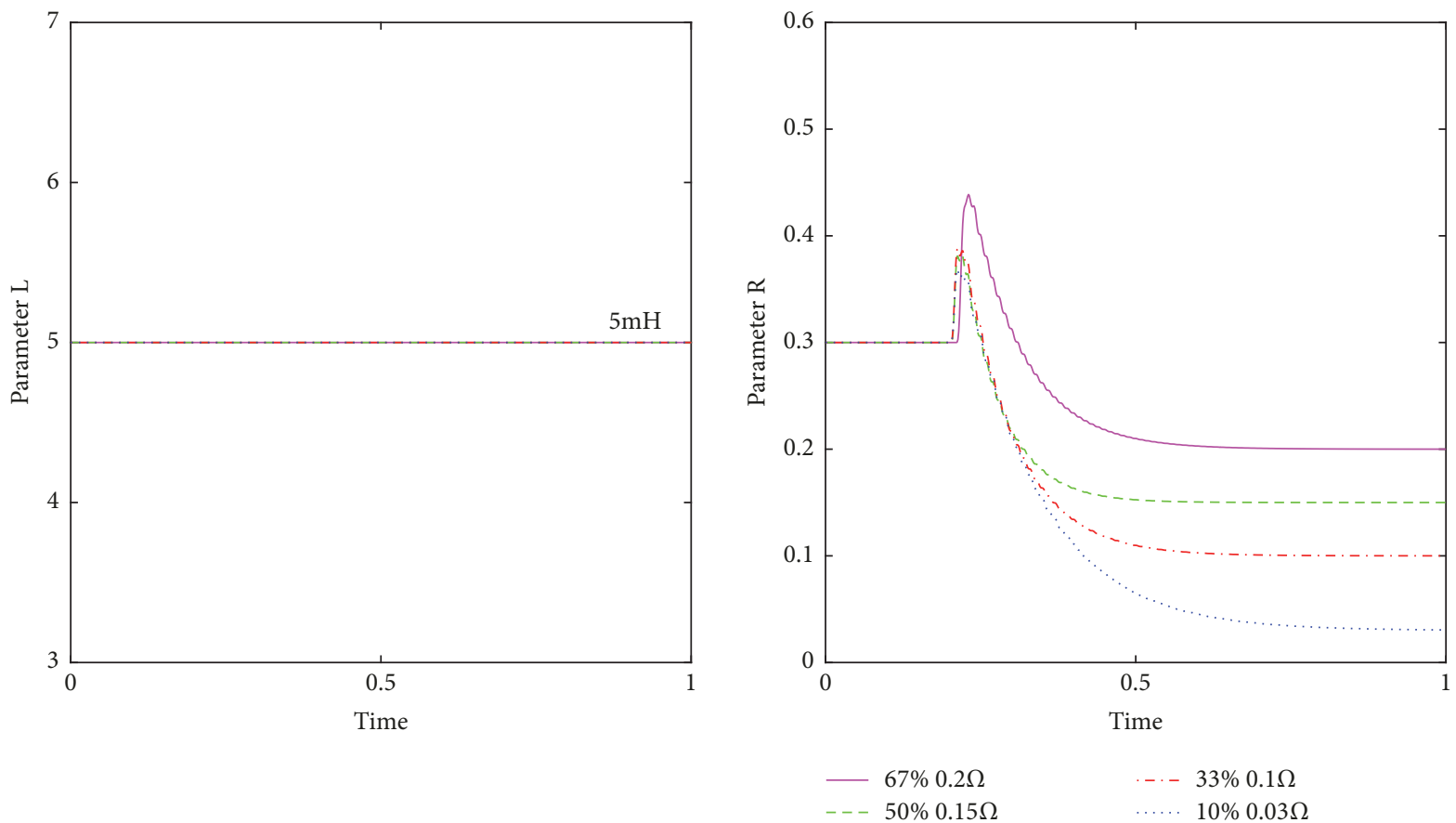

Figure 7: Parameter identification as $R$ changes from $67 \%$ to $10 \%$.

on linear-switched, are designed by using principles of robust. Through the outputs of these observers, the fault can be detected and identified accurately. Moreover, the proposed adaptive parameter identification for a single phase transformerless inverter is developed digitally and validated. In the last section, the simulation results show accurate fault diagnose and fast convergence speed for parameter identification. 

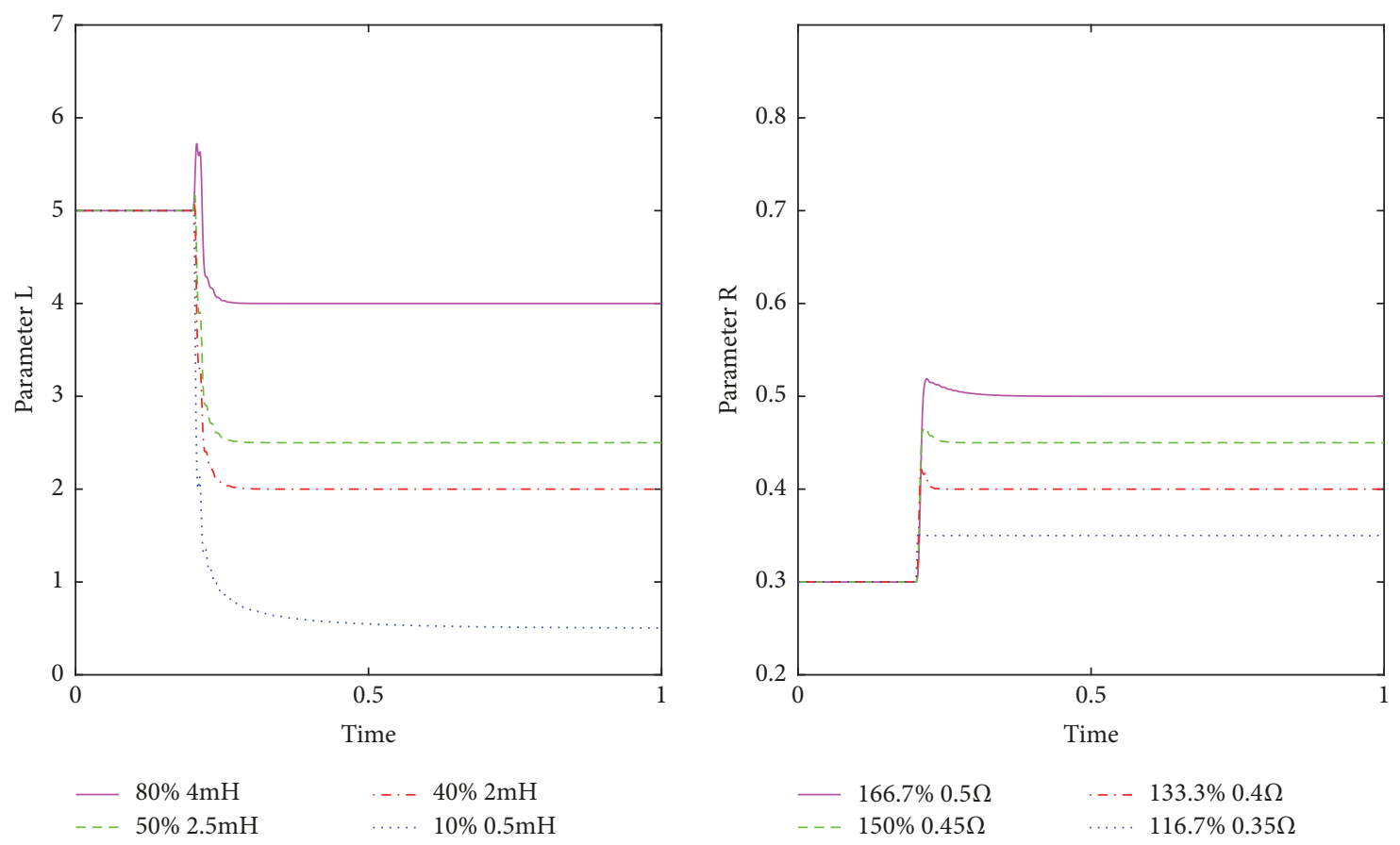

Figure 8: Parameter identification as $L$ changes from $67 \%$ to $10 \%$ and $R$ changes from $166.7 \%$ to $116.7 \%$.

\section{Data Availability}

The results simulations are based on MATLAB; readers can access all the original data, including parameters and programs, by sending an email to Dr. Bo Li at libo@neepu.edu.cn.

\section{Conflicts of Interest}

The authors declare that they have no conflicts of interest.

\section{Acknowledgments}

This work was supported by the National Natural Science Foundation of China [Grant nos. 61503071 and 61703091]; the Natural Science Foundation of Jilin Province [Grant no. 20180520211JH]; and the Science Research of Education Department of Jilin Province [Grant nos. 201693 and JJKH20170106KJ].

\section{References}

[1] W. Jiang, C. Dong, E. Niu, and Q. Wang, "Observer-Based Robust Fault Detection Filter Design and Optimization for Networked Control Systems," Mathematical Problems in Engineering, vol. 2015, Article ID 231749, 11 pages, 2015.

[2] X. Ding, J. Poon, and I. Celanovic, "Fault detection and isolation filters for three-phase AC-DC power electronics systems," IEEE Transactions on Circuits and Systems I: Regular Papers, vol. 60, no. 4, pp. 1038-1051, 2013.

[3] M. Kaufhold, H. Auinger, M. Berth, J. Speck, and M. Eberhardt, "Electrical stress and failure mechanism of the winding insulation in PWM-inverter-fed low-voltage induction motors," IEEE Transactions on Industrial Electronics, vol. 47, no. 2, pp. 396-402, 2000.
[4] S. Yang, A. Bryant, P. Mawby, D. Xiang, L. Ran, and P. Tavner, "An industry-based survey of reliability in power electronic converters," IEEE Transactions on Industry Applications, vol. 47, no. 3, pp. 1441-1451, 2011.

[5] H. Wang, M. Liserre, and F. Blaabjerg, "Toward reliable power electronics: Challenges, design tools, and opportunities," IEEE Industrial Electronics Magazine, vol. 7, no. 2, pp. 17-26, 2013.

[6] S. Nie, X. Pei, Y. Chen, and Y. Kang, "Fault diagnosis of PWM DC-DC converters based on magnetic component voltages equation," IEEE Transactions on Power Electronics, vol. 29, no. 9, pp. 4978-4998, 2014.

[7] R.-J. Wai, Y.-F. Lin, and Y.-K. Liu, "Design of Adaptive FuzzyNeural-Network Control for a Single-Stage Boost Inverter," IEEE Transactions on Power Electronics, vol. 30, no. 12, pp. 72827298, 2015.

[8] W. U. Xu-Sheng and M. A. Wei-Ming, "Prameters part identification of double-winding ac-dc generators," in Proceedings of the Csee, 2005.

[9] H. Renaudineau, J.-P. Martin, B. Nahid-Mobarakeh, and S. Pierfederici, "DC-DC converters dynamic modeling with state observer-based parameter estimation," IEEE Transactions on Power Electronics, vol. 30, no. 6, pp. 3356-3363, 2015.

[10] G. E. Pitel and P. T. Krein, "Real-time system identification for load monitoring and transient handling of Dc-Dc supplies," in Proceedings of the PESC '08 - 39th IEEE Annual Power Electronics Specialists Conference, pp. 3807-3813, Greece, June 2008.

[11] A. Barkley and E. Santi, "Improved online identification of a DC-DC converter and its control loop gain using crosscorrelation methods," IEEE Transactions on Power Electronics, vol. 24, no. 8, pp. 2021-2031, 2009.

[12] C. E. Hann, I. Singh-Levett, B. L. Deam, J. B. Mander, and J. G. Chase, "Real-time system identification of a nonlinear fourstory steel frame structure-application to structural health 
monitoring," IEEE Sensors Journal, vol. 9, no. 11, pp. 1339-1346, 2009.

[13] M. B. Abadi, A. M. S. Mendes, and S. M. Â. Cruz, "Method to diagnose open-circuit faults in active power switches and clampdiodes of three-level neutral-point clamped inverters," IET Electric Power Applications, vol. 10, no. 7, pp. 623-632, 2016.

[14] H. Ma and F. Ying, "Investigation on compatible remote fault diagnosis system for power electronic equipments," in Proceedings of the 5th International Conference on Power Electronics and Drive Systems, PEDS 2003, vol. 1, pp. 630-635, Singapore, November 2003.

[15] S. Kwak, U.-C. Moon, and J.-C. Park, "Predictive-control-based direct power control with an adaptive parameter identification technique for improved AFE performance," IEEE Transactions on Power Electronics, vol. 29, no. 11, pp. 6178-6187, 2014.

[16] S. Kouro, P. Cortés, R. Vargas, U. Ammann, and J. Rodríguez, "Model predictive control-a simple and powerful method to control power converters," IEEE Transactions on Industrial Electronics, vol. 56, no. 6, pp. 1826-1838, 2009.

[17] Z. Cen and P. Stewart, "Condition parameter estimation for photovoltaic buck converters based on adaptive model observers," IEEE Transactions on Reliability, vol. 1, no. 13, p. 99, 2017.

[18] J. Poon and S. R. Sanders, "Analysis and design of an adaptive parameter estimator for power electronics circuits," in Proceedings of the 18th IEEE Workshop on Control and Modeling for Power Electronics, COMPEL 2017, pp. 1-7, July 2017.

[19] L. A. Kamas and S. R. Sanders, "Parameter and State Estimation in Power Electronic Circuits," IEEE Transactions on Circuits and Systems I: Fundamental Theory and Applications, vol. 40, no. 12, pp. 920-928, 1993.

[20] J. Boaventura Cunha, C. Couto, and A. Ruano, "Real-Time Parameter Estimation of Dynamic Temperature and Humidity Models for Greenhouse Adaptive Climate Control," IFAC Proceedings Volumes, vol. 30, no. 26, pp. 49-54, 1997.

[21] M. Ahmeid, M. Armstrong, S. Gadoue, M. Algreer, and P. Missailidis, "Real-Time Parameter Estimation of DC-DC Converters using a Self-Tuned Kalman Filter," IEEE Transactions on Power Electronics, vol. 1, no. 1, p. 99, 2016.

[22] R. Dixon and A. W. Pike, "Application of condition monitoring to an electromechanical actuator: a parameter estimation based approach," Computing and Control Engineering Journal, vol. 13, no. 2, pp. 71-81, 2002.

[23] A. N. Manikas and L. F. Turner, "Adaptive signal parameter estimation and classification technique," IEE Proceedings $F$ Radar and Signal Processing, vol. 138, no. 3, pp. 267-277, 1991.

[24] Z. Mustapa, S. Saat, S. H. Husin, and T. Zaid, "Quadcopter physical parameter identification and altitude system analysis," in Proceedings of the 2014 IEEE Symposium on Industrial Electronics and Applications, ISIEA 2014, pp. 130-135, Malaysia, October 2014.

[25] S. K. Gudey and R. Gupta, "Second order sliding mode control for a single phase voltage source inverter," in Proceedings of the 2014 IEEE Region 10 Conference, TENCON 2014, Thailand, October 2014.

[26] A. B. Youssef, S. K. El Khil, and I. Slama-Belkhodja, "State observer-based sensor fault detection and isolation, and fault tolerant control of a single-phase PWM rectifier for electric railway traction," IEEE Transactions on Power Electronics, vol. 28, no. 12, pp. 5842-5853, 2013.

[27] J. Poon, P. Jain, C. Spanos, S. K. Panda, and S. R. Sanders, "Fault Prognosis for Power Electronics Systems Using Adaptive
Parameter Identification," IEEE Transactions on Industry Applications, vol. 53, no. 3, pp. 2862-2870, 2017.

[28] Y. Li, G. He, and J. Li, "Nonlinear robust observer-based fault detection for networked suspension control system of maglev train," Mathematical Problems in Engineering, vol. 2013, Article ID 713560, 7 pages, 2013. 


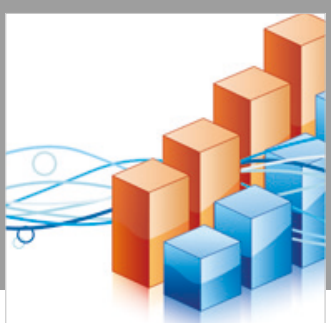

Advances in

Operations Research

\section{-n-m}
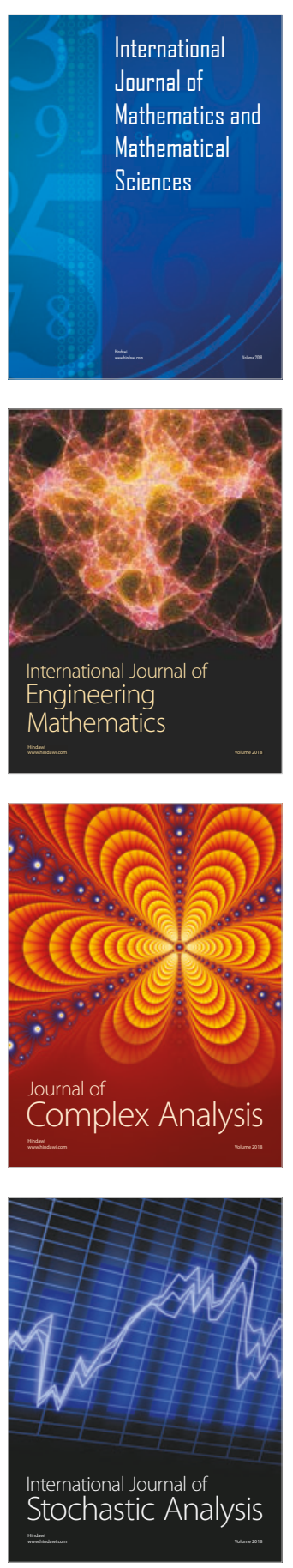
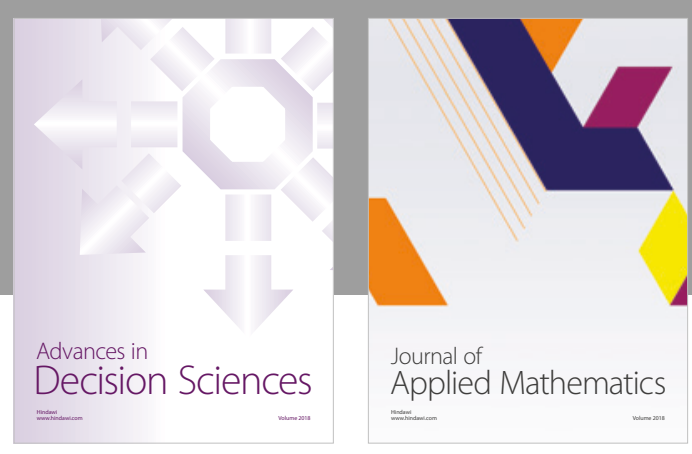

Journal of

Applied Mathematics
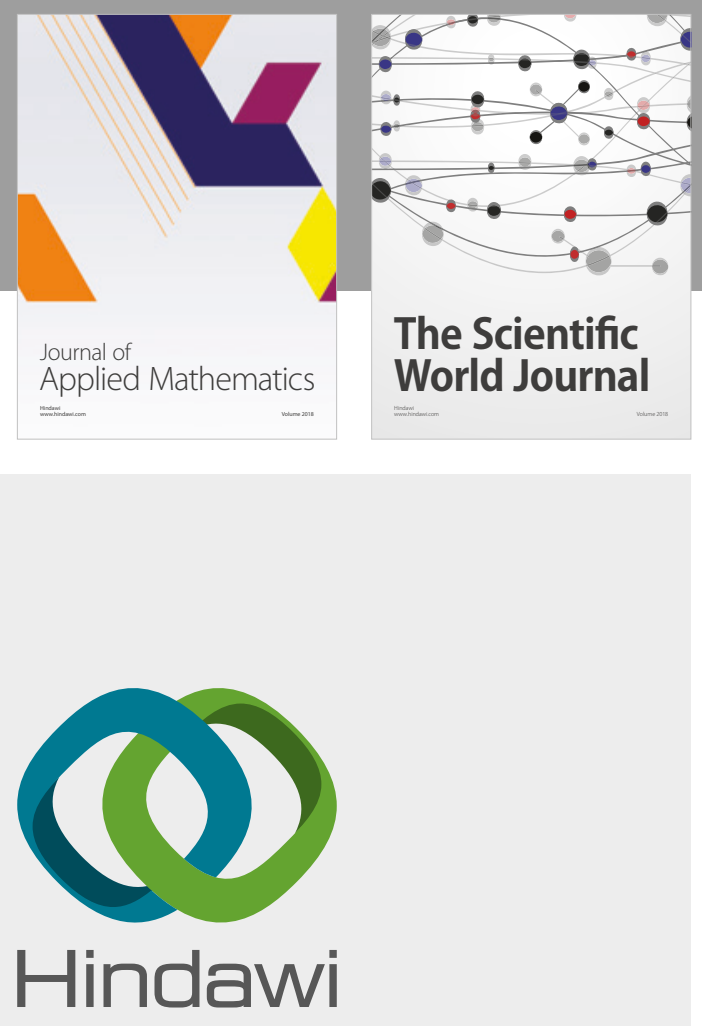

Submit your manuscripts at

www.hindawi.com

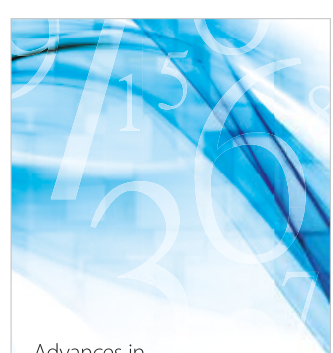

Advances in
Numerical Analysis
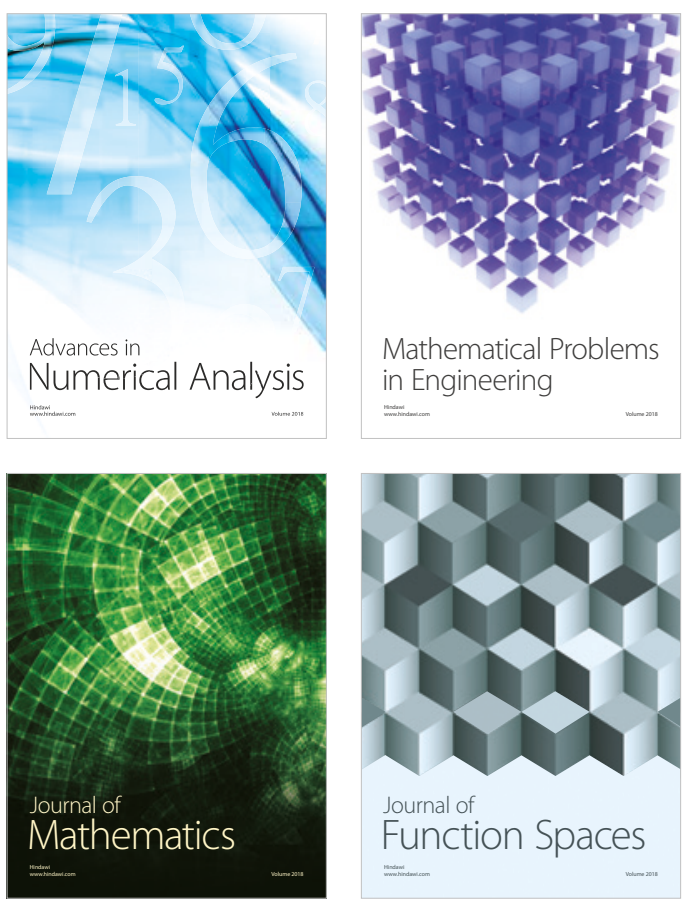

Mathematical Problems in Engineering

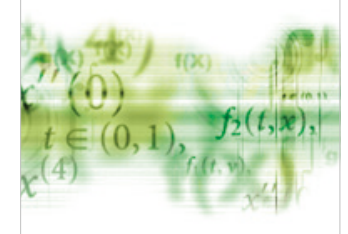

International Journal of

Differential Equations

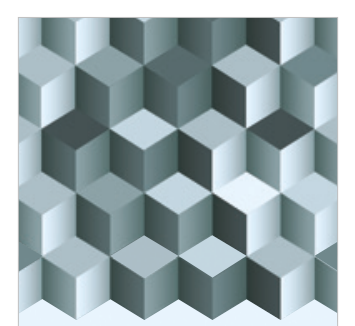

Journal of

Function Spaces

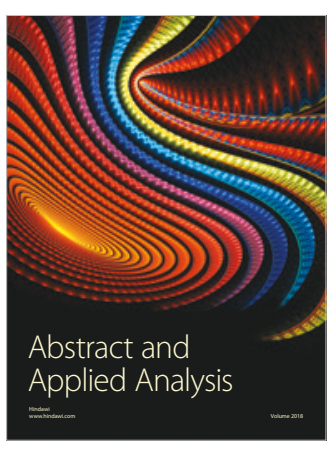

The Scientific

World Journal

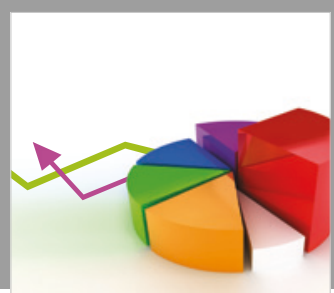

Journal of

Probability and Statistics
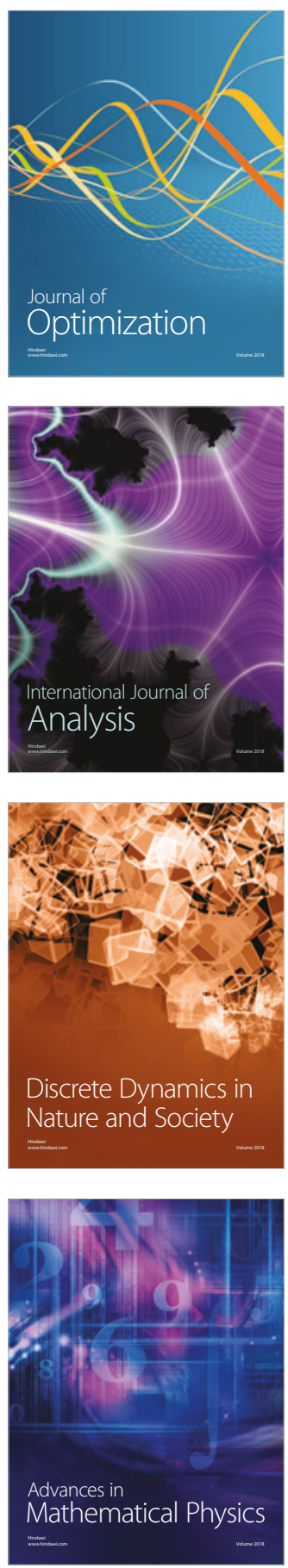